\title{
Archéopages
}

Archéopages

Archéologie et société

39 | 10/2013-01/2014

Le sort des vaincus

\section{Les victimes de la bataille du Mans (12-13 décembre 1793). Apports archéo-anthropologiques}

\section{Élodie Cabot}

\section{(2) OpenEdition}

\section{Journals}

Édition électronique

URL : https://journals.openedition.org/archeopages/540

DOI : 10.4000/archeopages. 540

ISSN : 2269-9872

\section{Éditeur}

INRAP - Institut national de recherches archéologiques préventives

\section{Édition imprimée}

Date de publication : 1 septembre 2014

Pagination : 32-39

ISSN : $1622-8545$

\section{Référence électronique}

Élodie Cabot, «Les victimes de la bataille du Mans (12-13 décembre 1793). Apports archéo-

anthropologiques », Archéopages [En ligne], 39 | 10/2013-01/2014, mis en ligne le 01 janvier 2016, consulté le 21 janvier 2022. URL : http://journals.openedition.org/archeopages/540 ; DOI : https:// doi.org/10.4000/archeopages.540 


\section{Les victimes de la bataille du Mans \\ (12-13 décembre 1793) Apports archéo-anthropologiques}

Élodie Cabot Inrap, UMR 7268-ADés-Anthropologie bio-culturelle, Droit, Éthique \& Santé

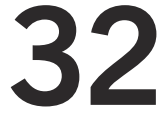

1. Pour exemple, la fouille de la tombe d'AlainFournier (Adam, 2006) les recherches conduites sur les lignes de front de la guerre 1914-1918 (Desfossés, Jacques, Prilaux, 2008), ou encore les travaux sur la retraite de Russie menés à Vilnius (Signoli et al., 2008).
Le contexte politique et social de la fin du XVIII ${ }^{\mathrm{e}}$ siècle après l'instauration de la république et l'exécution du roi Louis XVI le 21 janvier 1793 est explosif. La France, « pays régicide », affronte une coalition armée de l'Europe monarchique. À ces fronts de guerre aux frontières, s'ajoute sur son sol le soulèvement des populations de l'Ouest initié à Cholet début mars en réaction au décret de réquisition du 25 janvier organisant la levée de 300 ooo hommes afin d'augmenter les effectifs de l'armée républicaine. Ce mouvement populaire, vite réprimé au nord de la Loire, donne naissance à la Chouannerie, une guérilla qui touche la Bretagne et le Maine. Au sud du fleuve, dans ce qui est appelé la Vendée militaire, les attroupements de jeunes hommes s'étoffent et s'organisent. Pour Dieu et le Roi, des effectifs considérables sont mobilisés dans ce qui prendra le nom d'Armée catholique et royale [ill. 1] (Martin, 2007 ; Meyer-Sablé, Le Corre, 2007). Les guerres de Vendée font partie d'une histoire récente qui, dans l'ouest de la France, imprègne encore les mentalités : cet épisode reste très controversé dans la littérature, et les débats entre les «artisans inconditionnels de la Révolution » et les « héritiers des Vendéens » sont vifs (Gérard, 2013). La terminologie employée par les historiens en témoigne : " génocide, mémoricide, déni de mémoire...» (Sécher, 2011 ; Gérard, 2013). Des associations alimentent la mémoire des événements (Souvenir Vendéen, Virée de Galerne, Souvenir Chouan...) et des politiques s'invitent dans le débat à l'exemple d'une proposition de loi « relative à la reconnaissance du génocide Vendéen de 1793-1794 », soumise plusieurs fois par des députés. Aux textes et aux débats d'idées, nous pouvons maintenant proposer une approche archéoanthropologique de l'un des faits majeurs des guerres de Vendée : la bataille du Mans, lieu emblématique de la défaite des Vendéens les 12-13 décembre 1793.

En 2010, la fouille préventive menée place des Jacobins au Mans - qui a succédé à deux diagnostics réalisés en 1999 et 2009 (Chevet, 1999 ; Chevet, Cabot, Le Boulaire, 2009 ; Cabot, Chevet, Schmitt, 2009 ; Cabot, Chevet, Duchesne, 2012) - met au jour des fosses ayant servi à l'inhumation des victimes du conflit entre républicains et royalistes dans la ville (Chevet, Cabot, à paraitre 2015). Les périodes récentes bénéficient de plus en plus d'une approche interdisciplinaire intégrant l'archéologie, l'anthropologie et d'autres spécialités ${ }^{\mathbf{1}}$; la fouille des fosses révolutionnaires du Mans s'inscrit dans ces nouvelles études dédiées à la compréhension des événements d'un passé proche.

\section{Le contexte historique}

La bataille du Mans conclut l'expédition militaire connue sous le nom de Virée de Galerne qui voit l'armée royaliste traverser la Loire en direction du nord. Cette campagne a pour objectif la prise d'un port en eaux profondes afin d'assurer le débarquement de troupes anglaises et, avec ce soutien, rétablir le pouvoir monarchique (Dupuy, 1997 ; Gréau, 2012). La remontée au nord se solde par un échec devant Granville en novembre. Larmée épuisée va alors chercher à rentrer en Vendée le plus vite possible. Les insurgés échouent à passer la Loire à Angers les 4 et 5 décembre. Talonnés par l'armée républicaine, ils remontent au Mans qu'ils prennent sans difficulté le 1o décembre afin d'y puiser de nouvelles forces. À l'arrivée au Mans, l'armée royaliste n'est plus formée que de 30 à 40 ooo personnes sur les 80 ooo de départ, dont seulement 10 à 15000 combattants. Le reste de la troupe est composé de blessés, de femmes et d'enfants. La population du Mans, quant à elle, est estimée à 18115 habitants lors du recensement 


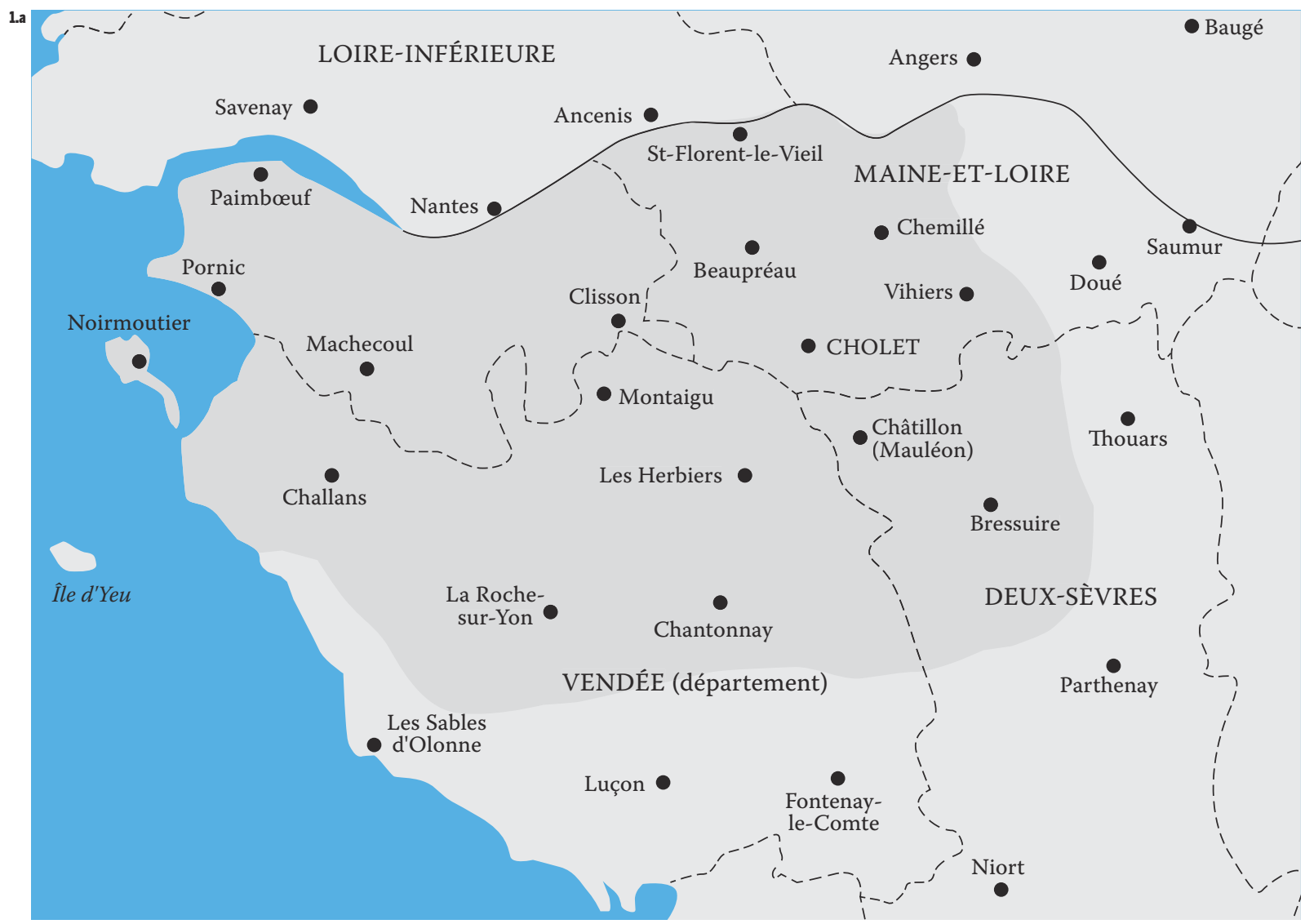

1.a. Des effectifs

considérables sont

mobilisés dans de

véritables batailles

rangées et, pendant le

printemps 1793, les

succès militaires de

I'Armée catholique et

royale s'enchainnent. Ces

premières victoires sont

rendues possibles par la

soudaineté du

mouvement populaire

mais aussi par le manque

de réaction des autorités

républicaines face à ce

qui s'avère plus

important qu'un

mouvement éphémère.

L'échec devant Nantes Ie

29 juin marque le déclin

du soulèvement,

cruellement mis à mal par

l'armée de Mayence

devant Cholet le

17 octobre.

La Vendée Militaire

(territoire insurgé en 1793)

$1 . \mathrm{b}$

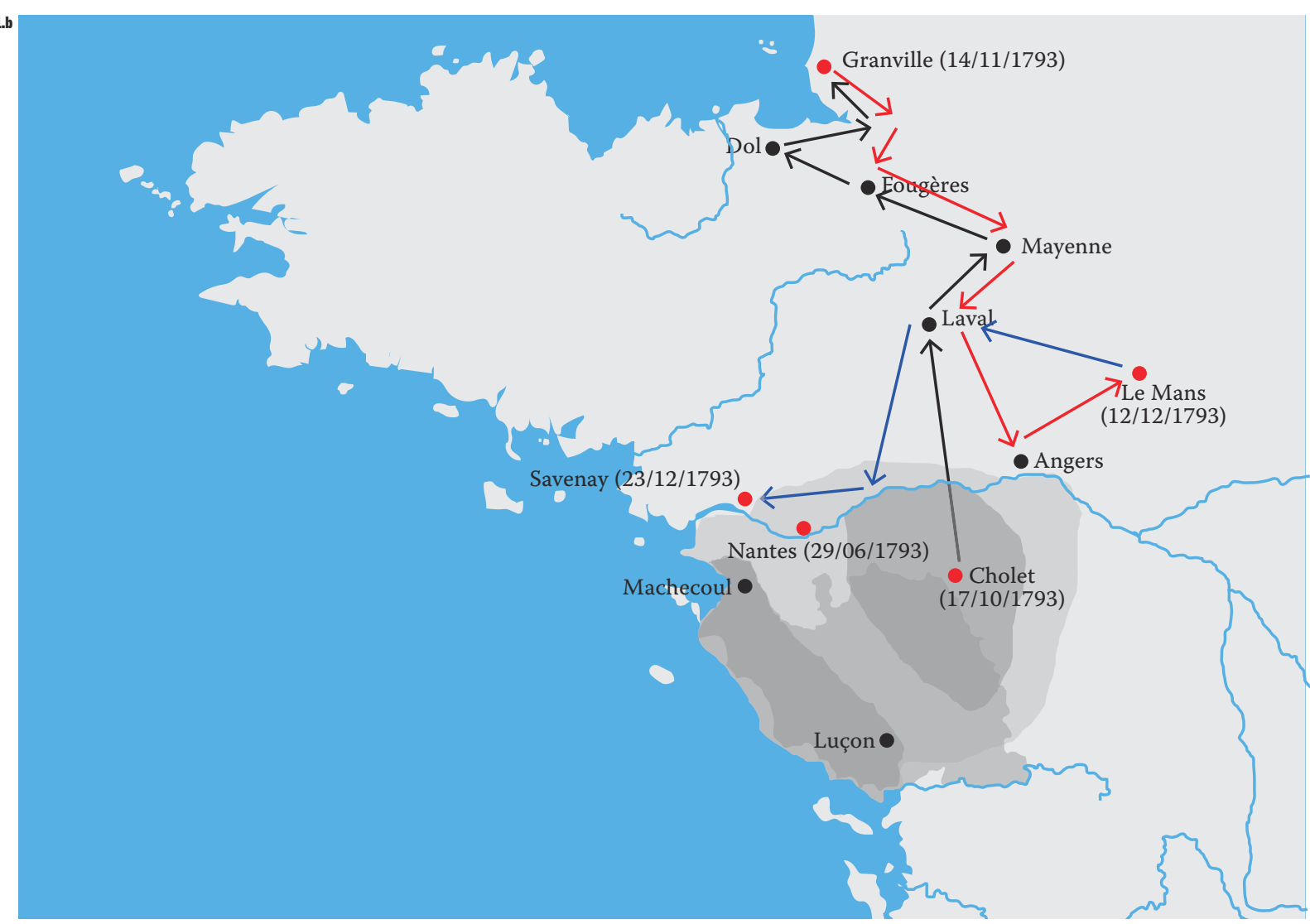

1.b. La défaite de Cholet marque le début d'une longue errance de l'Armée catholique et royale, qui ne compte plus que 60 à 80000 personnes dont une majorité de non-

combattants (femmes, enfants, vieillards). Ils sont placés sous le commandement de La Rochejacquelein, jeune généralissime de 21 ans, et décident de repasser la Loire afin de chercher du soutien auprès des

Anglais. Cette expédition, nommée Virée de Galerne, doit son nom au vent dominant soufflant du nord-ouest. La remontée au nord se solde par l'échec devant Granville les 14 et 15 novembre. La défaite de Savenay clôture définitivement cette expédition.
Zone de troubles épisodiques Principales zones d'insurrection Actuel département de la Vendée

- Principales défaites vendéennes
La Virée de Galerne

$\longrightarrow$ la montée vers Granville

$\longrightarrow$ la descente vers Le Mans

$\longrightarrow$ le repli vers Savenay 

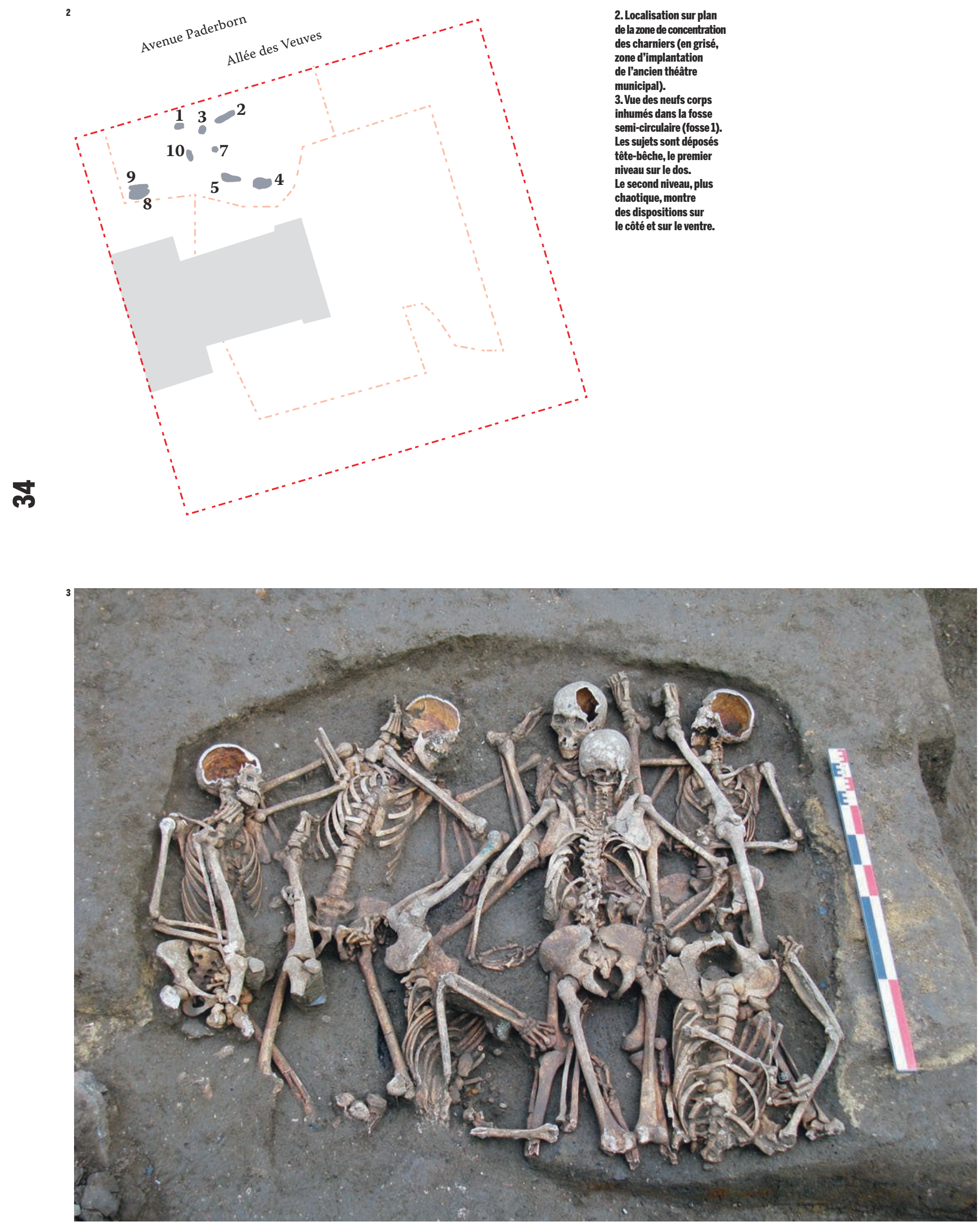
de septembre 1793. En face, l'armée républicaine est forte de 20 à 30000 militaires aguerris. Ces derniers reprennent la ville à partir du 12 décembre et les combats dans le centre durent jusqu'au 13 au matin. La topographie urbaine - notamment létroitesse des rues - entrave la fuite des Vendéens : l'estimation des victimes montre le déséquilibre des forces en présence avec environ 100 décès républicains pour 2500 à 3000 morts côté vendéen dans la souricière mancelle. Le bilan total est alourdi par la poursuite des royalistes sur la route de Laval et les historiens s'accordent sur un total de 10 à 15 ooo morts. La Virée de Galerne s'achève le 23 décembre 1793, avec la destruction quasi totale du reste de l'Armée catholique et royale à Savenay (Loire-Atlantique).

À la suite des combats des 12 et 13 décembre, les rues du Mans sont jonchées de cadavres. L'armée républicaine lancée à la poursuite des fuyards et les élites municipales locales ayant fui à l'arrivée des Vendéens (dès le 1o décembre, ils reviennent le 27 frimaire soit le 17 décembre), ce sont les habitants restés dans les murs pendant les troubles qui prennent en charge l'enlèvement et l'enfouissement des corps.

Si la littérature contemporaine ou postérieure aux faits (Bonchamps, 2003 ; Kléber, 2008; La Rochejaquelein, 1993 ; Savary, 1824 ; Westermann, 1794 ; Chardon, 1869-1872 ; Launay, 2006 ; Sécher, 1986 et 2011 ; Martin, 2007 et 2013 ; Dupuy, 1997 ; Gérard, 2013) abonde sur le déroulement des guerres de Vendée, la Virée de Galerne, les combats et les exactions réelles ou fantasmées sur les cadavres, la gestion de l'inhumation des corps sur les terrains de bataille reste peu abordée. Au Mans, seul le rapport du bureau de la Commission des arts publié en 1798, soit cinq ans après les faits, en témoigne. On y apprend que l'enlèvement des cadavres, effectué tout d'abord de manière anarchique, s'organise dès le 14 décembre, avec la nomination d'une administration provisoire chargée des inhumations. Ainsi les corps jonchant les rues du Mans furent ensevelis en deux jours dans de "grandes fosses » en «divers endroits ». Le quinconce des Jacobins, terrain public appartenant autrefois aux monastères des Cordeliers et des Jacobins, fut l'un des lieux choisis. Le ramassage dans la ville s'effectua à l'aide de 95 tombereaux permettant le transport de 20 à 22 corps à la fois. Aucun traitement différentiel entre les victimes républicaines, vendéennes ou encore civiles n'est indiqué. En 1815, les fosses des Jacobins sont de nouveau mentionnées lors du projet de nivellement de l'avenue Paderborn (au nord de la place) dans le registre de délibérations du conseil municipal du Mans (séance du 21 novembre 1815) : des « cavités hideuses » auraient fait l'objet d'un réaménagement avec adjonction de chaux. En 1960 encore, de nombreux témoignages oraux évoquaient les ossements, affleurant dans l'allée bordant le nord de la place, communément nommée «allée des veuves » en souvenir des inhumations. Enfin, en 1982, des restes osseux sont découverts lors de la création de toilettes publiques au nord-ouest de la place.
Au lendemain de ces violents combats, le nombre absolu de morts, mais aussi le contexte des combats ne permettent pas une gestion classique des inhumations. Un tel pic de mortalité en un temps court est généralement issu d'épisodes épidémiques, de catastrophes naturelles ou encore de conflits armés. Dans ces contextes, l'urgence conduit souvent à procéder à des inhumations multiples dans des fosses de grandes dimensions, de même qu'un seul lieu est généralement choisi pour les enfouissements. Des différences s'observent toutefois dans le traitement des cadavres en fonction des contextes de crises. Dans les situations de conflits armés, les corps sont principalement enterrés, voire abandonnés près ou sur les champs de bataille (Signoli, 2008) et parfois regroupés et inhumés ultérieurement dans des ossuaires (Rigeade, 2007 et 2008). Au Mans, ce ne sont pas les armées - ni les vainqueurs, ni encore moins les vaincus qui vont gérer l'enlèvement des corps, mais les habitants abandonnés par les autorités municipales. On peut penser qu'ils ont agi sous la crainte d'une propagation de maladies liées à la putréfaction, peut-être augmentée par la réputation de cette armée décrite comme l'armée des gueux ou des pouilleux. À cela pourrait s'ajouter une volonté d'oublier l'événement au plus vite.

\section{Les données archéologiques, analyses taphonomique et anthropologique}

Neuf fosses contenant 159 corps ont fait l'objet d'une fouille exhaustive de mai à juillet 2010 [ill. 2] ${ }^{2}$. Les travaux d'analyse et de recherche pluridisciplinaires étant encore en cours, les résultats communiqués ici sont susceptibles dêtre légèrement modifiés à l'issue des études ${ }^{3}$. Cependant, certains éléments permettent déjà de dégager des axes de réflexion. À considérer que les individus mis au jour sont exclusivement issus de l'Armée catholique et royale, les 159 sujets découverts représenteraient 5 à $7 \%$ du total des victimes (entre 2500 et 3 ooo victimes intra muros, cf. supra). On ne peut cependant pas écarter l'hypothèse que de rares civils ou encore des républicains dépouillés aient été inhumés en même temps. Cependant, la proportion inégale des victimes des deux camps ainsi que la forte présence de femmes dans les fosses incitent à penser que ce lieu d'inhumation comporte une majorité de royalistes.

L'étude taphonomique témoigne de l'urgence des ensevelissements et de leur prise en charge par la population. Huit creusements sont de forme rectangulaire. Une unique fosse s'écarte de ce schéma par sa forme semi-circulaire [ill. 3]. Les tranchées rectangulaires sont de dimensions variables et mesurent pour la plus grande jusquà quatre mètres de longueur. Le nombre de corps déposés varie de 3 à 53 ; l'épaisseur du dépôt atteint un mètre pour la fosse la plus importante. Ces tranchées sont condamnées par de la chaux en couverture totale avec des épaisseurs atteignant æzz30 cm ou par des dépôts sporadiques témoignant d'un manque de matière première. Aucune trace de réintervention 
4. Relevé des 53 sujets

inhumés dans le charnier 5

et du mobilier isolé lors

de la fouille (balles de

fusil, boutons, boulets

biscaïens, mitraille....).

Les corps apparaissent

pêle-mêle, dans la

position de réception

dans la fosse.

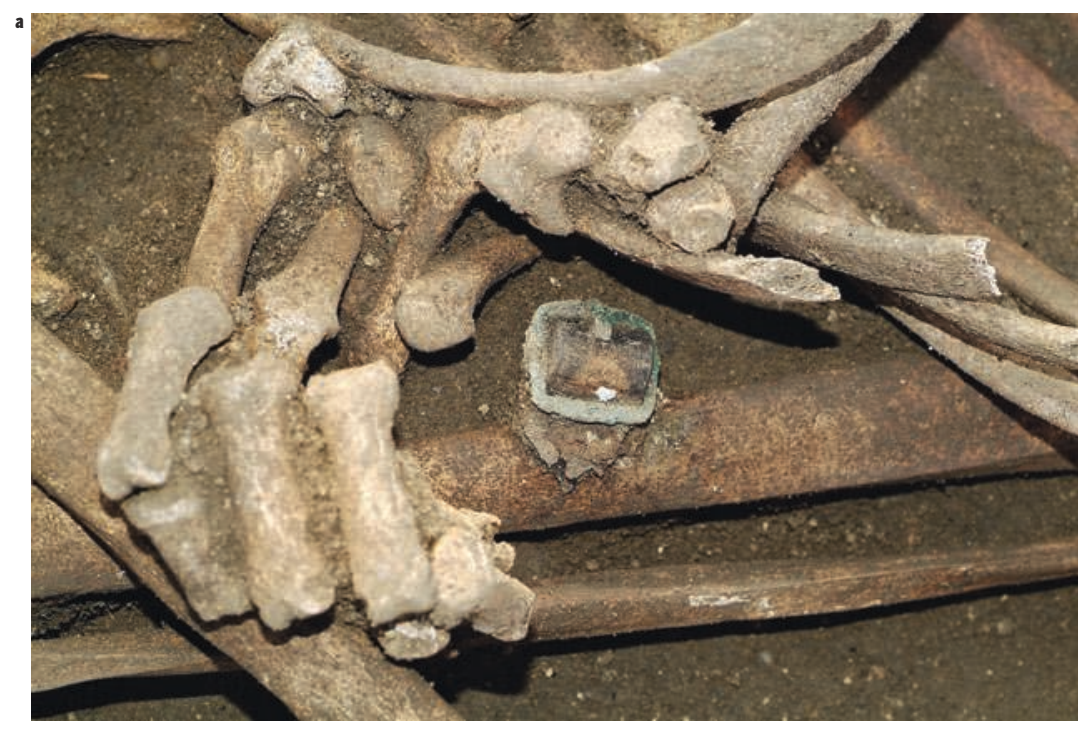

5.a. Boucle et reste

de cuir, attache de botte

ou de guêtre sur le tibia

de l'individu 7, sujet

masculin, charnier 1.

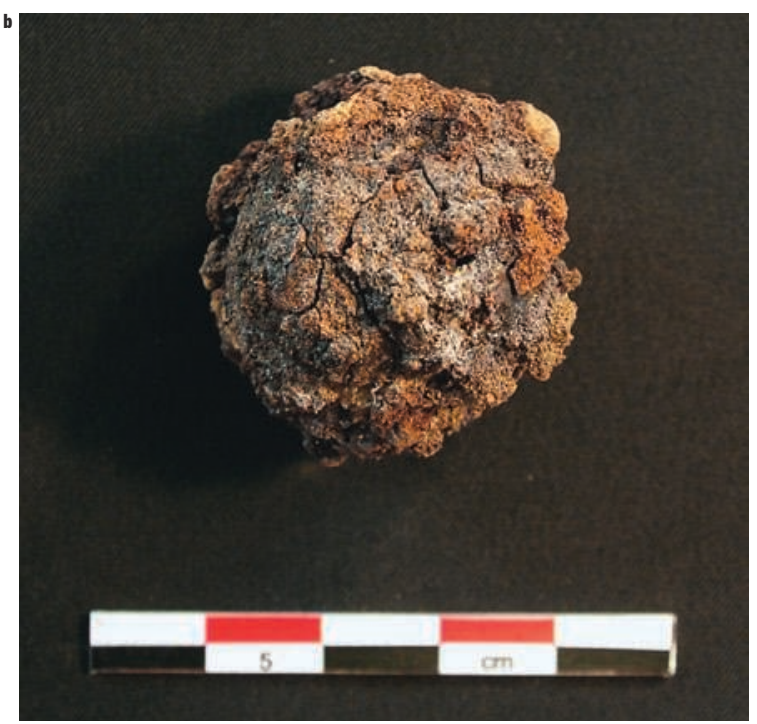

5.b. Boulet biscaïen retrouvé sous le fémur gauche du sujet 519,

individu de sexe féminin, charnier 5 . 
n’a été mise en évidence sur les restes osseux : on ne peut confirmer les recharges en chaux des « cavités hideuses » mentionnées dans les délibérations du conseil municipal de 1815 . Les corps sont basculés du tombereau dans les fosses, par les côtés latéraux des tranchées. Ils apparaissent pêle-mêle, sur le dos, le ventre ou encore le côté, les membres enchevêtrés, dans la position de réception dans la fosse [ill. 4].

L'analyse du mobilier présent dans les tombes indique que les corps ne sont pas complètement dépouillés et dénudés [ill. 5]. En effet, des boutons civils, mais aussi militaires, ainsi que des objets personnels sont encore portés. Les boutons, généralement en bois ou en os, sont pour certains recouverts d'une fine capsule de métal témoignant de l'appartenance à un régiment militaire républicain (hussard, dragon, grenadier). Les rares exemplaires de boutons militaires ne correspondent jamais à un costume complet (vestes, culotte...) et sont probablement issus de récupérations sur des cadavres lors de la longue Virée de Galerne. Les boutons civils correspondent pour certains à des chemises ou encore des fermetures de culotte bouffante au niveau des mollets. Une boucle métallique, accompagnée d'un lambeau de cuir et surement associée à une botte ou des guêtres, était encore en position sur la cheville gauche d'un sujet du charnier 1. Les objets personnels sont rares et de faible valeur commerciale, ils témoignent soit de la vie quotidienne (couteau, petite monnaie, épingles), soit d'une appartenance religieuse (médaille, croix...). Les objets de prix ont probablement été récupérés mais sans systématisme. Des monnaies en argent (écus Louis XV) ou encore une croix dorée ont été retrouvées dans les fosses, elles avaient probablement été dissimulées dans les vêtements. La chaux versée directement sur les cadavres des niveaux supérieurs a parfois conservé l'empreinte de membres (une épaule, un genou...), mais aussi des traces de tissus dont la trame des fibres est encore lisible et permet d'infirmer l'hypothèse d'un dénudement complet des corps.

L'unique fosse semi-circulaire, non condamnée par de la chaux, contient les dépouilles partiellement dénudées de neuf sujets disposés tête-bêche. D'aucuns ont cru voir dans cette différence de traitement funéraire une sépulture réservée aux soldats républicains qui auraient ainsi bénéficié d'une attention particulière malgré l'urgence des ensevelissements. Cette question de la différenciation des armées lors des inhumations est difficile, voire impossible à déterminer sur les restes osseux. Cependant, la présence d'une femme parmi ces neuf adultes infirme l'hypothèse d'un recrutement strictement militaire. Par ailleurs, des boutons représentant les corps d'armée républicains ont été retrouvés en faible nombre dans les fosses, mais aucun dans celle-ci.

La détermination sexuelle a été menée selon trois procédures : d'abord une analyse morphométrique à partir des os coxaux (DSP, Murail et al., 2005) complétée par une étude statistique à partir des mesures enregistrées sur le squelette post-crânien ${ }^{4}$ (DSS, Murail, 1996 ; Murail et al., 1999) puis une détermination du sexe moléculaire à partir de l'ADN fossile permettant notamment de sexer les jeunes adolescents $^{\mathbf{5}}$. Ainsi, plus de $90 \%$ de l'échantillon a pu être sexé. On observe une représentation importante de femmes (1/3 de l'effectif) qui confirme les textes et leur description de cette armée, composée de jeunes hommes mais aussi de femmes. Les adultes sont jeunes, majoritairement âgés de moins de trente ans, peu d'entre eux portent de traces de sénescence (Schmitt, 2005). Pour les enfants (Scheuer, Black, 2000 ; Schaefer, Black, Scheuer, 2008), les données biologiques montrent une proportion d'immatures de $12,5 \%$, pour la plupart des adolescents socialement assimilables à de jeunes adultes ayant de fait pris part aux combats. Seuls deux enfants de moins de cinq ans ont été identifiés. Les jeunes enfants accompagnant l'armée «blanche » ne se retrouvent pas dans cet échantillon archéologique.

On observe une discrimination sexuelle significative dans cinq fosses. $\mathrm{Si}$, pour quatre charniers, les sujets des deux sexes sont représentés dans des proportions proches, pour les cinq autres, les hommes sont majoritaires dans trois fosses (charniers 5, 9 et 10) et les femmes dans deux (charniers 7 et 8) [ill. 6].

Ces regroupements « sexués » dans les fosses ne semblent pas volontaires, mais reflètent plus probablement la dynamique du ramassage des corps, effectué par quartier urbain, révélant ainsi différentes phases de la bataille (combats rapprochés ici, embuscades ou charges de cavalerie là, canonnades et fuites ailleurs), ayant impliqué des populations distinctes. En effet, l'analyse de la représentation des projectiles retrouvés dans les fosses, la typologie des blessures par l'étude comparée de leur répartition et leur caractérisation (arme blanche/arme à feu, choc frontal/choc postérieur...), avec l'identification biologique (homme/femme, adulte/adolescent), même si elle reste trop incomplète à ce jour pour avancer des chiffres fiables, permettent toutefois de proposer cette hypothèse. La majorité des projectiles issus de fusils ou de pistolets de même que les boulets biscaïens ont été retrouvés dans les charniers 5 et 9 majoritairement masculins, ils sont accompagnés de nombreux éléments métalliques associés à de la mitraille. Dans certaines fosses (charnier 8 par exemple, majoritairement féminin) seules des traces de coups tranchants ont été notées, assénées sur le crâne ou les jambes, ces coups sont principalement portés d'en haut et par l'arrière. Ces différences, pressenties à l'étude, semblent indiquer deux aspects des combats : d'un côté, deux armées face à face et, de l'autre, une masse de non-combattants poursuivie lors de sa fuite hors les murs.

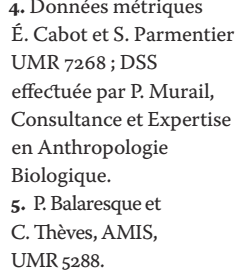

Biologique.

5. P. Balaresque et

C. Thèves, AMIS, 




6. Vue des charniers 8 et 9 en cours de fouille. La fosse 8 (en haut) ne renferme que des sujets de sexe féminin (21 sujets) décédés pour la plupart des suites de coups portés à l'arme blanche (sabre majoritairement). La fosse 9 est exclusivement

\section{masculine (13 sujets) \\ mais renferme toutefois \\ un jeune enfant âgé de \\ 3 à 5 ans. Les corps sont \\ pour certains criblés \\ de balles, et d'autres \\ présentent des \\ traumatismes liés à \\ l'emploi d'arme blanche.}

\section{Références bibliographiques}

Bonchamps M. M. R. de, 2003, Mémoires de Madame la marquise de Bonchamps sur la Vendée, rédigés par la comtesse de Genlis, (1 ${ }^{\mathrm{re}}$ éd. 1823), Cholet, Pays et Terroirs, $136 \mathrm{p}$.

Commission centrale de correspondance des Arts, « Rapport des événements relatifs à l'arrivée de l'armée vendéenne dans le département de la Sarthe au mois de Xbre 1793 », 16 août 1798, copie manuscrite par Négrier de la Crochardière Médiathèque du Mans, manuscrit $\mathrm{B} 21 \mathrm{~A}$, version microfilmée MI 396.

KLÉBER J.-B., 2008, Mémoires politiques et militaires 1793-1794, (1 ${ }^{\text {re }}$ éd. 1907), Cholet, éd. Pays et Terroirs, 2 vol., 376 et $230 \mathrm{p}$.

La Rocheja Quelein (Marquise de) 1993, Mémoires de madame la marquise de La Rochejaquelein, ( $\mathrm{r}^{\mathrm{re}}$ éd. Paris, Michaud, éditeur du roi, 1815), Cholet, Pays et Terroirs, $616 \mathrm{p}$.

LAUnAy A., 2006, La Terreur en Anjou, correspondance et journal de Benaben, commissaire civil en Maine et-Loire auprès des armées républicaines, $\left({ }^{\mathrm{re}}\right.$ éd. 1886), Cholet, Pays et Terroirs, 483 p.

SAVARY J.-J.-M., 1824, Guerres des Vendéens et des chouans contre la République française ou Annales des départements de l'Ouest pendant ces guerres par un officier supérieur de la République habitant dans la Vendée avant les troubles, Paris, Baudoin Frères, 516 p.

Westermann F.-J., 1794, Campagne de la Vendée du général de brigade Westermann, Paris, an II de la République une et indivisible, $42 \mathrm{p}$.

ADAM F., 2006, Alain-Fournier et ses compagnons d'armes. Une archéologie de la Grande Guerre, Metz, Ed. Serpenoise, 219 p.

Савот É., Chevet P., Duchesne S., 2012, La bataille $d u$ Mans, apports archéo anthropologiques à l'étude des guerres de Vendée, 10-13 décembre 1793, XXX Colloque GALF, Dakar (Antropo, 27), p. 15-22. www. didac.ehu.es/antropo

Cавот É., Chevet P., Schmitt L., 2009, « Les charniers des guerres de Vendée au Mans : approche archéoanthropologique », in Les ombres de l'Empire. Approches anthropologiques, archéologiques et historiques de la Grande Armée, CERMA ( $\mathrm{HS} \mathrm{n}^{\circ} 5$ ), p. 123-128.

Chardon H., 1869-1872, Les Vendéens dans la Sarthe, Le Mans, Imprimerie Edmond Monnoyer, 3 vol., xv-401, 327 et $483 \mathrm{p}$

Chevet P., 1999, Quinconce des Jacobins, Le Mans (Sarthe), sondages d'évaluation, rapport de diagnostic, Inrap-SRA Pays-de-la-Loire, non paginé.

Chevet P., САВот É., à paraître 2015, Quinconce des Jacobins, Le Mans (Sarthe), rapport d'opération, Inrap-SRA Pays-de-la-Loire.

Chevet P., CABot É., Le Boulaire C., 2009, Quinconce des Jacobins, Le Mans (Sarthe), rapport de diagnostic, Inrap-SRA Pays-de-la-Loire, $96 \mathrm{p}$.

DESEOSSÉS Y, JACQUES A. PRLLAUX G, 2008, L’Archéologie de la Grande Guerre, Rennes, éd. Ouest-France, Inrap (Coll. Histoire), $127 \mathrm{p}$.

Dupuy R., 1997, Les Chouans, Paris, Hachette Littératures (coll. La Vie Quotidienne), 287 .

Gérard A., 2013, Vendée, les archives de l'extermination, La Roche-sur-Yon, éd. du CVRH (Centre vendéen de recherches historiques), $692 \mathrm{p}$

GRÉAU P., 2012, La Virée de Galerne : 18 octobre-23 décembre 1793, Cholet, éd. Pays et Terroirs, 392 p.

Martin J.-C., 2007, La Vendée et la Révolution, Paris, éd. Perrin, (Coll. Tempus), $283 \mathrm{p}$

MARTIN J.-C., 2013, Un détail inutile ? Le dossier des peaux tannées, Vendée, 1794, Paris, Vendémiaire éd., 160 p.

Meyer-SABlé N., Le Corre C., 2007, La Chouannerie et les guerres de Vendée, Rennes, éd. Ouest-France (Coll. Histoire), $127 \mathrm{p}$
Murail P., 1996, Biologie et pratiques funéraires des populations d'époque historique: une démarche méthodologique appliquée à la nécropole galloromaine de Chantambre. Thèse de l'université de Bordeaux I, $267 \mathrm{p}$.

Murail P., BruzeK J., Braga J., 1999, « A new approach to sexual diagnosis in past populations. Practical adjustmenst from van Vark's procedure », International Journal of Osteoarchaeology, 9, p. 39-53.

Murail P., Bruzek J., Houet F., Cunha E, 2005, "A tool for probabilistic sex diagnosis using worlwide variability in hip bone measurements », Bulletins et Mémoires de la Sociétéd'Anthropologie de Paris, n.s., 17, 3-4, p. 167-176.

RIGEADE C., 2007, Les sépultures de catastrophe. Approche anthropologique des sites d'inhumations en relation avec des épidémies de peste, des massacres de population et des charniers militaires, Oxford, Archeopress (BAR International S1695) $129 \mathrm{p}$.

RIGEADE C., 2008, « Approche archéo-anthropologique des inhumations militaires », Socio-anthropologie [En ligne], 22 2008, mis en ligne le 14 octobre 2009, URL: http://socio-anthropologie.revues.org/1153

SCheuer L., Black S., 200o, Developmental Juvenile Osteology, Academic Press, 518 p.

SChaefer M., Black S., SCheuer L., 2008, Juvenile Osteology : a laboratory and Field manual, Amsterdam, Burlington, $384 \mathrm{p}$.

SCHMITT A., 2005, «Une nouvelle méthode pour estimer lâge au décès des adultes à partir de la surface sacropelvienne iliaque », Bulletins et Mémoires de la Société d'Anthropologie de Paris, n.s., t. 17, 2005, 1-2, p. 2-13.

SÉCHER R., 1986, La Vendée-Vengé: Le génocide francofrançais, Paris, PUF, $338 \mathrm{p}$.

SÉCHER R., 2011, Vendée. Du génocide au mémoricide. Mécanisme d'un crime légal contre l'humanité, Paris, éd. Le Cerf (coll. Cerf politique), $448 \mathrm{p}$

SignOLI M., 2008, « Archéo-anthropologie funéraire et épidémiologie », Socio-anthropologie [En ligne], 22 | 2008, mis en ligne le 14 octobre 2009, URL : http://socio-anthropologie.revues.org/1155

Signoli M. (dir.), Vette T., Dutour O., Ardagna Y. 2008, Vilna 1812-Vilnius 2002 Les oubliés de la retraite de Russie, Paris, Éditions Historiques Teissèdre, 176 p. 